Tejedor Tejedor, F.J. (2018). Investigación educativa: la utilidad como criterio social de calidad. Revista de Investigación Educativa, 36(2), 315-330.

DOI: http://dx.doi.org/10.6018/rie.36.2.326311

\title{
Investigación educativa: la utilidad como criterio social de calidad
}

\author{
Educational research: \\ utility as a social criterion of quality
}

Francisco Javier Tejedor Tejedor'

Universidad de Salamanca

\begin{abstract}
Resumen
La comunidad educativa en general considera que la investigación educativa no proporciona resultados útiles. Tratamos de reflexionar sobre los fundamentos de esta opinión intentando aportar propuestas que puedan aproximarnos a la consecución del objetivo que consideramos prioritario de la investigación educativa: aportar explicaciones razonables de los hechos o fenómenos estudiados a fin de contribuir a la creación de un cuerpo coherente de conocimientos, orientados a producir la información necesaria para mejorar la acción educativa. Pero no es fácil ponerse de acuerdo a la hora de concretar su finalidad ni de delimitar las características que debe tener esa investigación: para unos se trata de desarrollar el conocimiento sobre los procesos educativos en tanto que otros consideran prioritario la mejora de la práctica.

Palabras clave: Investigación educativa; criterios de calidad; innovación; utilidad; mejora de la acción educativa.
\end{abstract}

1 Mientras revisaba los últimos párrafos de este artículo me llega la noticia del fallecimiento del profesor Arturo de la Orden, guía, compañero y amigo durante muchos años. Sirvan estas líneas como reconocimiento a su extraordinaria labor académica y, por encima de todo, a su excelencia como persona.

Correspondencia: Francisco Javier Tejedor Tejedor, email tejedor@usal.es, C/ Faisanes, 8 S. Marta, 37900 Salamanca (España). 


\begin{abstract}
In general, the educational community considers the educational research does not provide useful results. We try to reflect on the basis of this opinion, trying to provide proposals in order to reach the objective that we consider prioritative in educational research: provide reasonable explanations of the facts or phenomena studied in order to contribute to the creation of a coherent body of knowledge, oriented to produce the necessary information to improve the educational practice. But it is not easy reach an agreement when we want to establish the true purpose of our research or delimit its characteristics: for some people it is about developing knowledge about educational processes while others consider the improvement of practice as a priority.

Keywords: educational research; quality criteria; innovation; utility; improvement of educational action.
\end{abstract}

\title{
¿Para qué la investigación educativa?
}

"Decir que la investigación educativa está desprestigiada o que no obtiene resultados útiles para la educación son dos expresiones categóricas que reflejan no sólo el sentir de quienes las enunciaron... sino de la comunidad educativa en general. La investigación y la práctica educativas van por caminos distintos, irreconciliables y casi opuestos, lo que hace que su colaboración se antoje muy complicada" (Perines y Murillo, 2017. p. 90).

Aceptamos esta opinión y tratamos de reflexionar sobre su razón de ser (Sabariego, 2004), para aportar propuestas que puedan posibilitar esa colaboración demandada y aproximarnos a la consecución del objetivo que consideramos prioritario de la investigación educativa: producir información necesaria para contribuir a la mejora de la acción educativa.

La investigación científica es un proceso que, mediante la aplicación del método científico, nos permite obtener información relevante y fidedigna para generar, verificar, corregir o aplicar el conocimiento. El objetivo básico de la ciencia es la teoría, que establece presuntas relaciones entre fenómenos naturales. La investigación es la verificación científica de la teoría, que pretende explicar los fenómenos observados.

La investigación educativa debe ser una actividad científica $\mathrm{y}$, por tanto, formal, sistemática, controlada, empírica y objetiva. Debe poseer los atributos del método científico. Su objetivo básico debe ser aportar explicaciones razonables de los hechos o fenómenos estudiados, a fin de contribuir a la creación de un cuerpo coherente de conocimientos, orientados a producir la información necesaria para mejorar la acción educativa (Tejedor, 2004; 2005).

Pero no es fácil ponerse de acuerdo a la hora de concretar su finalidad ni de delimitar las características que debe tener esa investigación: para unos se trata de desarrollar el conocimiento sobre los procesos educativos, en tanto que otros consideran prioritario la mejora de la práctica (De la Orden y Mafokozi, 1999; Díaz Costa, 2009). Esta escisión entre la teoría y la praxis es, opinan algunos, el más doloroso y lamentable fenómeno que se arrastra desde los inicios de la reflexión humana acerca de la educación. 
Quienes se esfuerzan por lograr la síntesis de posturas, tratarán de analizar científicamente lo que se viene haciendo por los prácticos de la educación; de transformar en "conocimiento científico" la experiencia de los prácticos. La consecución plena de este objetivo va a requerir la participación conjunta de unos y otros en las tareas de investigación, lo que supone concebir la investigación educativa como "un proyecto de acción", como "un programa de intervención", vinculada a las necesidades especificadas por los prácticos.

Algunos autores no establecen este debate en términos de científicos sociales (teóri$\cos$ ), por una parte, y docentes (prácticos), por otra; sino entre "actos de investigación" y "actos sustantivos". Un acto de investigación es un acto para impulsar una indagación. Un acto sustantivo se halla justificado por algún cambio en la realidad.

La investigación básica es un "acto de investigación"; difícilmente en la investigación básica pueden unirse el acto de investigación y el acto sustantivo. Sí que puede conseguirse esa unión en la investigación aplicada; en la investigación-acción el acto investigador es necesariamente un acto sustantivo.

Podemos aceptar entonces, de acuerdo con Carr y Kemmis (1988), que la misión de la investigación educativa consiste en generar teorías sustantivas que busquen su fundamento en las complejidades de la realidad práctica:

La teoría sólo adquiere la consideración de científica en tanto que sugiere maneras mejoradas de entender las experiencias educativas y sólo adquiere validez educativa en tanto que dichas sugerencias son puestas a prueba y confirmadas por la experiencia práctica... La teoría sólo adquiere un carácter educativo cuando ella misma es corregida, mejorada y aconsejada a la luz de sus consecuencias prácticas. Es la práctica la que determina el valor de cualquier teoría educativa... (Carr y Kemmis, 1988, p. 139).

Habermas mantiene, como elemento básico de su teoría de los "intereses constitutivos de los saberes" (la famosa trilogía de intereses técnicos, prácticos y emancipatorios), que el conocimiento nunca es producto de una mente ajena a las preocupaciones cotidianas; se constituye siempre en base a intereses que han ido desarrollándose a partir de necesidades naturales de la especie humana. Sin atender a necesidades y deseos (es decir, sin utilidad), la humanidad no hubiera tenido interés por desarrollar el conocimiento. La grandeza de la ciencia es su servidumbre (Habermas, 1984).

Creemos en esta necesidad de mayor interconexión entre la "ciencia" y la "ciencias sociales" pese a aceptar que estas no son ciencias de la misma naturaleza que la concebida por Popper, Khun, Lakatos... Serían más bien "disciplinas sociales", mezcla de conocimiento teórico y acción práctica que reclaman perspectivas propias, que le han de venir dadas por la investigación.

La estrecha relación entre el progreso científico y social fue ejemplificado por la conocida declaración de Lewin (1978): "no investigar sin acción y no actuar sin investigación". La investigación en la acción pretende contribuir tanto a los intereses prácticos de las personas como a los objetivos de la ciencia social, integrando una colaboración dentro de un marco ético mutuamente aceptable. 
Esta postura superadora caracteriza las ciencias de la educación como tecnologías, definidas como: "Un conjunto de conocimientos organizados, obtenidos de manera rigurosa, que ayudan a resolver con eficacia problemas de interpretación de la realidad" (Bunge, 1980). Estas tecnologías son ciencias aplicadas orientadas hacia la búsqueda de conocimientos útiles que intentan resolver la tradicional dicotomía entre teoría y práctica a través de un campo de conocimientos compatibles con la ciencia coetánea, situando la norma de validez de todo conocimiento en su practicidad, su utilidad. A partir de estos presupuestos podemos denominar "tecnociencia" a esta modalidad de ciencia coetánea caracterizada por una muy amplia interconexión entre los presupuestos científicos y tecnológicos, lo que equivale a demandar del conocimiento su ineludible proyección a la solución de problemas sociales.

Así pues, por investigación educativa entendemos el estudio de los métodos y procedimientos utilizados para obtener un conocimiento científico (una explicación y una comprensión) de los fenómenos educativos orientado a la solución de problemas educativos y sociales. Y en el mejor de los mundos posibles, ese conocimiento puede obtenerse por cualquiera de las tres modalidades de investigación científicamente acreditadas: básica, aplicada e investigación-acción.

Obsérvese que hacemos referencia a la investigación básica. Podemos encontrar opiniones que nos recuerdan el valor fundamental de la investigación básica, en todos los ámbitos del conocimiento, incluido el educativo. Mario Bunge, en su discurso de investidura como Doctor Honoris Causa por la Universidad de Salamanca, afirmaba:

La investigación básica consiste en la búsqueda de la verdad independientemente de su posible uso práctico... Alimenta a la técnica sin ser técnica, porque la técnica diseña medios para cambiar el mundo en lugar de estudiarlo [...]Es deseable fomentar la ciencia básica, no sólo para enriquecer la cultura sino también para nutrir la técnica" (Bunge, 2003, p. 2).

La inclusión entre las modalidades de investigación científicamente acreditadas de la investigación aplicada y la investigación-acción se debe sin duda, a la aportación, entre otros de Lewin (1978) y a la conceptualización más reciente de Gibbons y otros (1997) caracterizando las nuevas formas de producir conocimiento:

- Los problemas de investigación se plantean en un contexto de aplicación. El conocimiento ha de ser útil para alguien.

- El conocimiento ha de ser socialmente distribuido.

- Se contempla la posibilidad de aportaciones transdisciplinares para la construcción de un campo científico determinado.

- Se amplía el número de lugares potenciales en los que se puede crear conocimiento.

- La responsabilidad social impregna todo el proceso de producción del conocimiento. La interpretación y difusión de los resultados de investigación se proyectan hacia la solución de problemas y a la satisfacción de necesidades.

- Se utilizan nuevos criterios para evaluar la calidad de la producción científica, incorporando criterios relacionados con aspectos sociales, económicos, políticos,... 
La preocupación por los fundamentos teóricos que orientan la investigación activa se debió inicialmente a Lewin (1951). Los estudios sobre tipologías le van a permitir analizar los cambios producidos tras un proceso de intervención; su teoría surge de una experiencia práctica. La noción de investigación activa supone no sólo la concreción de una investigación aplicada, sino también un vínculo estrecho con la investigación fundamental. Este elemento activo de la investigación tiene reconocimiento empírico y puede ser utilizado con fines prácticos e intervencionistas. Para Lewin, investigación e intervención se apoyan mutuamente. La intervención sacará partido de la investigación en la medida en que ésta se extienda a la solución de problemas prácticos. La investigación activa se asimila, enriqueciéndola, a la noción de investigación aplicada, que en esencia supone:

- Admitir nuevas formas y espacios de creación de conocimiento.

- Una vez modificado el espacio escolar deberán modificarse las pautas metodológicas de investigación.

- Enfatizar el carácter singular de toda práctica.

- Incorporar nuevos modelos de programación (competencias, proyectos...).

Sugerencias para mejorar la investigación educativa

Presentamos nuestras opiniones sobre pautas de carácter metodológico que estamos convencidos pueden contribuir a la mejora de la calidad de los procesos y de la utilidad de la investigación educativa.

\section{Fundamentar teóricamente las investigaciones}

Posiblemente, la mayor deficiencia de la investigación educacional, que explica su escasa capacidad para contribuir a la solución de problema, es decir su utilidad, consiste en el hecho de que no se apoya en una teoría. Se actúa en el marco del "desnudo empirismo": se recogen datos mediante un cuestionario o una encuesta y se intenta encontrar una respuesta "observando" la información... Se obtienen muchos datos, pero se han hallado muy pocas respuestas; se obtienen muchos datos, pero no se prueba nada.

Desde planteamientos epistemológicos fundamentados se viene señalando ya desde hace tiempo que los fracasos de muchas investigaciones pedagógicas fueron erróneamente atribuidas al método (sobre todo al experimental o a cualquiera de sus afines) siendo así que la verdadera fuente de error era la inadecuación de la teoría que las sustentaba o la carencia de la misma (Dendaluce, 1988).

Al tener como referencia una teoría, el punto de partida del investigador es un problema definido en términos precisos y en un marco de supuestos formulados con claridad, de los que deduce hipótesis susceptibles que deberán ser verificadas. La teoría, así entendida, es en esencia "Un conjunto de supuestos a partir de los cuales pueden extraerse una serie de leyes (principios) empíricos" (Van Dalen y Meyer, 1994, p. 312).

Otros autores van más allá y no sólo reclaman la referencia a la teoría en el proceso de la investigación educativa, sino que opinan que su misión principal debe ser generar teorías sustantivas que busquen su fundamento en las complejidades de la realidad práctica: 
La teoría sólo adquiere la consideración de científica en tanto que sugiere maneras mejoradas de entender las experiencias educativas y sólo adquiere validez educativa en tanto que dichas sugerencias son puestas a prueba y confirmadas por la experiencia práctica... La teoría sólo adquiere un carácter educativo cuando ella misma es corregida, mejorada y aconsejada a la luz de sus consecuencias prácticas. Es la práctica la que determina el valor de cualquier teoría educativa... (Carr y Kemmis, 1988, p. 139)

La generación de una teoría sustantiva no es sólo el punto de llegada de un proceso sino el punto de partida del siguiente; ese es el mecanismo lógico del avance científico. Desde mi punto de vista este ciclo "teoría-hechos-investigación-teoría" no se ha producido en el contexto pedagógico y ello ha supuesto que la investigación se convierta en esfuerzos aislados para explicar hechos aislados; se pierde gran parte de su sentido al no entroncarse en una teoría que dé forma a los hallazgos habidos y que, al tiempo, genere nuevas ideas para seguir trabajando. Este planteamiento equivale a entender la investigación como un conjunto de actuaciones sucesivas orientadas a conceptualizar la realidad (la práctica). Para conseguir este objetivo puede seguirse un doble proceso:

a) De teorización: Este proceso empieza en los hechos. Es un proceso ascendente, inductivo. Sus elementos constitutivos serían los datos, la formación de proposiciones y la articulación de teorías.

b) De verificación: La investigación parte de la teoría y desciende a la realidad empírica. Los elementos fundamentales serían la teoría, los modelos, las hipótesis, la realidad y la verificación que relaciona las ideas y los hechos. Es un proceso descendente, hipotético-deductivo.

Me manifiesto, si cabe con más argumentos metodológicos de los expuestos hace años (Tejedor, 1988), partidario de la corriente de opinión epistemológica que potencia el valor de la teoría, bajo la afirmación de que cualquier actividad investigadora mejorará si obtiene sus premisas de los estudios teóricos. Y ello admitiendo que es la experiencia, la práctica, quien genera ideas a la investigación; pero desde un principio, el investigador debe situarse en un contexto teórico (la formulación de hipótesis debe suponerlo de hecho). La interpretación de los resultados de la investigación debe retomar, vía respuesta a la hipótesis, la conceptualización teórica.

\section{Adaptar la medición a presupuestos y contextos educativos}

Compartimos la opinión de Fernández Esquinas (2003, p. 47) cuando escribe:

En cualquier tipo de investigación científica un requerimiento fundamental es la calidad de los datos disponibles. Los datos son el fundamento de la evidencia científica, de la contrastación de hipótesis, de la generalización de los resultados de investigación y de la relevancia de las posibles teorías y marcos analíticos... Suele ser frecuente que en el ámbito de las ciencias sociales existan problemas derivados de la validez y de la fiabilidad de los datos que se pro- 
ducen y utilizan. En general, los datos para la investigación social son pobres en lo que se refiere al proceso de obtención y a los niveles de medición...

En la problemática que implica el tema de la medición educativa llevamos muchos años aplicando las pautas e instrumentos que nos llegan de ciencias "amigas", como la Psicología, que han dedicado muchos más esfuerzos y recursos que los educadores para ir mejorando progresivamente la calidad de la medida de todo tipo de variables relacionadas con los objetivos de la investigación. Considero, en términos generales, como muy elevada la calidad métrica de los instrumentos psicológicos a los que regularmente recurrimos. Por lo que mi primera recomendación a los investigadores educativos, para la medida de muchas de las variables que incorporamos a nuestras investigaciones, sea la de recurrir a la búsqueda de instrumentos disponibles (test, cuestionarios, escalas...) contrastados en sus características métricas (fiabilidad, validez...). La alternativa a esta opción es proceder a la elaboración propia de instrumentos, lo que supone un alto riesgo a la hora de valorar su calidad métrica y poner en cuestionamiento las posibilidades de referencia comparativas entre los resultados obtenidos por distintos investigadores.

Esta opinión en modo alguno es incompatible con el reconocimiento de la pertinencia de medidas procedentes de la aplicación de instrumentos que previamente requieran procesos de adaptación a determinados contextos o a datos procedentes de procesos de observación. Datos de observación extraordinariamente valiosos para la ciencia al emerger de los hechos, de la realidad. Pero datos que respondan a los requerimientos básicos de su potencial científico: ser controlada, o sea, ser objetiva y comprobable, empleando procedimientos y técnicas que eliminen al máximo las posibles fuentes de distorsión y error y estableciendo las condiciones bajo las cuales se ha observado el hecho.

Pero el uso aconsejable de instrumentos de medida de paternidad psicológica no puede hacernos olvidar la necesidad, en muchas ocasiones, de tener en cuenta la razón de ser del proceso de medición que realizamos, ya que no hacerlo nos llevaría a caer en lo que denominamos "trampa psicométrica". Evitar caer en esta trama consiste en considerar las diferentes finalidades que puede tener, en determinadas ocasiones, la medida psicológica y la medida educativa. En términos generales, podemos decir que la medida psicológica se plantea como objetivos fundamentales la diferenciación, recurriendo para ello a estímulos e ítems que favorezcan dicha diferenciación, para lo que tienen que responder a requisitos de tipo estadístico que favorezcan su poder discriminativo. Pero, en muchos casos, el objetivo de la medida educativa reside más en la verificación de los efectos de un proceso de intervención o formación que en la diferenciación; reside más en la verificación de la consecución de los objetivos o competencias. Por ello, desde hace años, se proyectó esta modalidad de medida educativa hacia modelos de referencia criterial, más que normativos.

Una última reflexión sobre una práctica habitual en la investigación educativa: el uso de cuestionarios como instrumento principal para la obtención de datos, en relación con otros como escalas de actitudes o entrevistas. Los cuestionarios proporcionan opiniones subjetivas de los sujetos a quienes de los aplicamos (profesores, alumnos, gestores...). Desde mi punto de vista, sería un gran avance en la calidad de la investiga- 
ción el intento de aproximarnos a la medida de conductas reales, a través de estrategias (registros) de observación o pruebas de "saber hacer". En temas como la evaluación de competencias, por ejemplo, nos proporcionaría datos muchos más reales que los que obtenemos por opinión de los encuestados; datos más reales con mayor potencial para orientar los procesos formativos consecuentes.

\section{Potenciar el papel del diseño de investigación}

Hace ya muchos años que Campbell y Stanley escribían:

El método experimental es el único medio de zanjar las disputas relativas a la práctica educacional, única forma de verificar adelantos en el campo pedagógico y único método para acumular un saber al cual pueden introducírsele mejoras sin correr el riesgo de que se descarten caprichosamente los conocimientos ya adquiridos a cambio de novedades de inferior calidad (Campbell y Stanley, 1978, p. 11).

Desde hace tiempo (Tejedor, 1994), y mucho menos en los tiempos actuales, ni entendiendo el término "experimental" en su concepción menos rigurosa (diseños cuasiexperimentales, experimentos de campo, pre-experimentales...) podría considerarse aceptable la opinión de los autores citados. Por suerte para la investigación, especialmente en el ámbito de las ciencias sociales, se extienden las posibilidades de llevar a cabo investigación "científica" ampliando el conjunto de estrategias, todas ellas debiendo responder a esquemas de planificación estructuradas; o sea, ajustándose a un determinado diseño o plan de trabajo.

El diseño de una investigación equivale a la secuencia completa de pasos fijados de antemano para asegurar que los datos requeridos se obtendrán de modo que posibiliten el análisis objetivo que nos conduzca a deducciones válidas respecto al problema de estudio. El propósito de cualquier diseño es proporcionar la mayor cantidad de información respecto al problema a investigar.

Para mejorar su eficiencia el diseño habrá de ser tan simple como sea posible: el buen diseño es condición imprescindible para un análisis riguroso; es posible ejecutar un estudio bien diseñado y analizar incorrectamente los datos resultantes, pero es imposible disponer de un diseño pobre y realizar un análisis excelente.

El diseño, por tanto, implica planificar las distintas etapas del proceso de investigación, lo que usualmente entendemos como "metodología". Esta metodología debe, lógicamente, ajustarse a las finalidades propuestas por el investigador. Creemos que un elevado porcentaje de las investigaciones educativas se interesan principalmente por describir la realidad, algunas intentan explicarla y muy pocas las que plantean cambiarla, es decir, aportar sugerencias para mejorar la acción educativa.

En términos generales, y pudiendo aplicarse a los distintos tipos de investigación (CIDE, 2001), puede afirmarse que la metodología utilizada se ha caracterizado por:

- Su sencillez, rayando en muchos casos la ingenuidad y la simpleza (meramente descriptiva). 
- Se abusa de algunas técnicas (encuesta, cuestionario, entrevista) como soporte único.

- Análisis de datos parcialmente inadecuados a los planteamientos de la investigación.

- Escasa proyección de los resultados a la solución de problemas.

Por nuestra parte destacamos la importancia de lo relacionado con el análisis de datos. Sugerimos:

- Evitar la "cuantofrenia" (datos por datos, tablas por tablas...)

- Seleccionar el programa de análisis, general o específico, adecuado (cuantitativo o cualitativo)

- Seleccionar la técnica de análisis que permita responder al objetivo o pregunta planteados

- Considerar que las pruebas estadísticas son robustas. Los incumplimientos leves de las condiciones de aplicación no suelen tener consecuencias graves

- Y tener muy en cuenta que ninguna prueba mejora la calidad del dato con el que llevamos a cabo el análisis.

\section{Considerar el papel de las nuevas tecnologías}

Desde hace algunos años las TIC han cambiado muchas cosas. En lo referente a la investigación educativa escribía hace unos años la conveniencia de su incorporación a las distintas tareas que implica el proceso investigador:

El Consejo Europeo celebrado en Liubliana en 2008 definió una nueva visión del espacio Europeo de Investigación basada en la libre circulación del conocimiento ("la quinta libertad"). Se destacaba la importancia de desarrollar infraestructuras que permitieran crear y divulgar la ciencia por medios electrónicos ("e-ciencia" y "e-investigación). La "e-ciencia" se refiere al uso de las TIC en la investigación científica; implica una tecnología de vanguardia, un poder de cómputo alto y fuentes de datos en una vasta red de entorno distribuido. La "e-investigación" es un término más amplio; se refiere a las formas en que los investigadores usan las TIC, en aproximación a los planteamientos previstos en la "e-ciencia". Por ello es necesario que las TIC estén integradas en todas las fases del proceso científico, tanto en lo referente a la creación del conocimiento como a su difusión (Tejedor y García-Valcárcel, 2012. p. 8).

En esta ocasión me interesa destacar cuatro aspectos que el uso de las TIC puede contribuir a mejorar la calidad de la investigación educativa:

- Incorporándolas como estrategia de recogida de la información, fundamentalmente dirigidas a la modalidad no presencial, que se puede gestionar a través del correo electrónico, los foros, audio o videoconferencias, formularios online, blogs, documentos online, registros audio o videográficos..., favoreciendo la rapidez en el acceso a la información facilitada por los participantes, así como la generación de los ficheros de datos para su tratamiento posterior.

- Analizando su efecto sobre la medida de las variables estudiadas. El trabajo educativo en entornos informáticos modifica muchas de las pautas que consideramos tradicionales (dimensión cognitiva, motivación, aprendizaje, trabajo 
colaborativo...). La medición-evaluación de esas variables requiere un esfuerzo añadido al investigador para responder a los nuevos usos y tratamientos de las prácticas didácticas. Es un importante reto que tenemos pendientes: podemos estar convencidos de la bondad del buen uso de las TIC pero no hemos generado las estrategias para evaluar esas bondades.

- Seleccionar adecuadamente el programa estadístico para el análisis de datos.

- Aprovechar todas las ventajas de la difusión de los resultados de la investigación.

\section{Realizar un esfuerzo de racionalización y de simplificación al diseñar el pro- yecto y de reconceptualización al final del proceso}

La investigación educativa está en una situación que exige, después de la fase de entusiasmo y de ensayo sin orden de todas las técnicas, un esfuerzo de racionalización y de simplificación. La racionalización puede considerarse un valor añadido de la fundamentación teórica que hemos señalado en comentarios anteriores.

La simplificación ha sido igualmente recomendada al indicar que la mejora de la eficiencia del diseño requiere que sea tan simple como sea posible. Podemos estar de acuerdo en considerar pensar que la educación es un fenómeno complejo, lo que no indica necesariamente estudiar en cada caso toda la problemática que encierra. Considero no solo posible sino conveniente fragmentar los problemas a investigar. Los modelos que guían la acción investigadora deben responder al principio científico de "parsimonia": contener el menor número posible de elementos que puedan proporcionar la información suficiente para explicar y comprender el fenómeno estudiado.

La reconceptualización hace referencia a la necesaria conexión de los resultados obtenidos con los problemas inicialmente planteados, lo que puede llevarnos, por una parte, a la confirmación o rechazo de los considerandos teóricos estudiados y, por otra, a la incorporación de innovaciones probadas en la práctica educativa.

\section{Indagar sobre los procesos de intervención}

La investigación educativa debe proponerse la superación de lo que podemos denominar "fase descriptiva" y orientarse hacia la obtención de información que contribuya a la mejora de los procesos de intervención, lo que desde un punto de vista paradigmático significa conectar la investigación a los proyectos de innovación (investigación-acción) y, desde el punto de vista metodológico, requiere la incorporación a los proyectos de variables susceptibles de ser modificadas por los procesos de intervención. Facilitaríamos así la concreción de los marcos teóricos de los procesos de enseñanza-aprendizaje.

Es preciso un tipo de investigación unida a proyectos de innovación que incluya la participación de profesores e investigadores en el marco de una política curricular. La investigación debe estar fundamentalmente pensada para transformar las condiciones existentes de la acción educativa, pensada para mejorar la educación. Hay que cerrar el proceso $\mathrm{I}+\mathrm{D}+\mathrm{i}$ : investigar para crear algo nuevo, probarlo e investigar de nuevo su incidencia en los cambios (Alonso, 2005). 
Desde hace tiempo conocemos las dificultades para integrar en la práctica educativa los resultados de la investigación (Stenhouse, 1991). El docente no percibe que las temáticas de investigación que responden al modelo "central-periferia" le resuelvan los problemas. Si queremos aumentar el impacto positivo de la investigación en el aula, es necesario asignar al profesor un papel activo en el diseño, desarrollo y evaluación del currículo y adoptar nuevas consideraciones: fomentar la investigación-acción, caracterizar al centro docente como unidad de investigación y fortalecer la colaboración entre los centros escolares y los centros de producción investigadora. Los temas que se traten en esta investigación responden lógicamente a los intereses de los centros y profesores implicados. Pero puede haber otros temas objeto de investigación que abordamos en el apartado siguiente.

\section{Investigación para responder a instancias socio-políticas}

La investigación educativa tiene también otras responsabilidades que atender. Me refiero a investigar sobre contenidos vinculados a la consecución de objetivos sugeridos desde las instancias socio-políticas y a los nuevos requerimientos científicos. Veamos algunas de esas demandas.

a) Objetivos educativos europeos y españoles. Estratégica educación y formación (fijados en el 2013 e integrados en el horizonte 2020) (MECD, 2013)

1. Hacer una realidad el aprendizaje a lo largo de la vida y la movilidad

2. Mejorar la calidad y la eficiencia de la educación y la formación:

- mejorar la formación de los profesores

- desarrollar aptitudes para la sociedad del conocimiento

- garantizar el acceso de todos a las TIC

- aprovechar al máximo los recursos

3. Promover la equidad, la cohesión social y la ciudadanía activa

- entornos de aprendizaje abierto

- hacer el aprendizaje más atractivo

- igualdad de oportunidades y cohesión social

4. Afianzar la creatividad y la innovación, incluyendo el espíritu emprendedor

- definir competencias y capacidades básicas en la nueva sociedad del conocimiento

- reforzar los lazos de la investigación con la vida laboral

- mejorar el aprendizaje de idiomas extranjeros

- formar en competencias digitales a niños, y adultos

- aumentar la movilidad y los intercambios para reforzar la cooperación europea

b) Crear escuelas que preparen para el futuro: nuevos escenarios, nuevas metodologías (Debats d'Educació -UOC, 2013). Para responder a preguntas tales como:

- ¿Qué lugar debe ocupar el aula en el proceso de aprendizaje del siglo XXI?

- ¿Cómo deben organizarse los centros educativos para crear contextos de aprendizaje reales?

- ¿Qué se puede aprender de los centros eficaces? 
- ¿Qué condiciones permitirán a los centros aprender y trabajar en comunidad?

- ¿Cómo integrar adecuadamente las TIC en los procesos de enseñanzaaprendizaje?

- ¿Qué competencias deben ser prioritarias?

- ¿Cómo habrá que trabajar los aprendizajes en un mundo tecnológico y globalizado?

\section{Comunicación comprensiva y formas diversificadas de difusión}

Queremos destacar la importancia de elaborar un buen informe final del proceso investigador, redactado bajo el presupuesto básico de responder a las exigencias de una comunicación comprensiva para la diversidad de colectivos a los que pueda ir dirigido, que recoja fielmente el proyecto inicial (objetivos y aspectos metodológicos), los procedimientos utilizados para la obtención de información, los resultados obtenidos, una valoración de los mismos ajustada a la consecución de los objetivos planteados y las propuestas pertinentes para orientar los cambios que puedan sugerirse.

Su elaboración y presentación debe suponer una importante interacción y reflexión con los equipos de los centros de los que se ha obtenido la información.

Su difusión deberá sobrepasar las instancias y las motivaciones de referentes como JCR y SJR y proponerse que puedan llegar, sobre todo a través de la Red, a los centros educativos (gestores, profesores, alumnos y padres) y a los colectivos implicados en las tareas docentes, especialmente a quienes muestren más interés por las actividades de innovación.

\section{Gestión del conocimiento: estrategias social y metodológicamente útiles}

\section{Formación del profesorado}

Si queremos que los profesores de los centros educativos incorporen entre sus funciones ordinarias las tareas de investigación es evidente que debemos potenciar su formación en aspectos metodológicos, especialmente los relacionados con metodologías de corte cualitativo. Habría que orientar estas políticas de formación en dos direcciones:

Mejorar la formación inicial del profesorado incorporando la metodología de investigación en los estudios de Grado de Infantil y Primaria y en el Máster de Secundaria.

Promocionar e incentivar la formación continua fundamentada en la revisión de estrategias y prácticas innovadoras que se consideren interesantes, ofreciendo a los profesores en activo la posibilidad de realizar cursos de iniciación a la investigación y ofreciendo asesoramiento metodológico a grupos de profesores que lleven adelante proyectos de innovación. Incluso entendiendo la innovación desde la óptica social y política, se acepta la planificación del cambio a partir de los resultados de la investigación. Parece conveniente llegar a mantener un equilibrio entre las actividades de desarrollo del currículo y la investigación. 


\section{Reconocer todos los tipos de investigación como potencialmente útiles}

Apoyamos el pluralismo integrador de las diferentes metodologías. Abogamos por la complementariedad metodológica. El retraso que se está produciendo en conseguir la confluencia metodológica en el ámbito educativo es el más claro indicador del permanente estado de cuestionamiento científico de nuestro quehacer. La falta de convergencia metodológica está dificultando el avance de la Pedagogía en su conformación científica y está produciendo por añadidura cada día un mayor alto grado de dependencia respecto a otras ciencias (psicología, sociología...).

Las posturas que preconizan la "ruptura" se fundamentan, unas, en la descalificación de resultados por la carencia de rigor en las estrategias básicamente subjetivas de obtención de información y, otras, en la negación del determinismo, en la no aceptación de aproximaciones explicativas simples a fenómenos complejos y en el cuestionamiento permanente de la objetividad (Clark, 1994). Creo que, ambas posturas son, por intransigentes, desafortunadas.

\section{Formación de equipos}

Cada día se considera más necesaria, casi obligada, la formación de equipos, que deberán concretarse en la realización de trabajos interdisciplinares y colaborativos intercentros (con buen uso de las redes).

Es preciso un tipo de investigación unida a proyectos de innovación que incluya la participación de profesores e investigadores en el marco de una política curricular. La investigación debe estar fundamentalmente pensada para transformar las condiciones existentes de la acción educativa, pensada para mejorar la educación. Hay que proponerse que la investigación permita cerrar el proceso I+D+i: investigar para crear algo nuevo, probarlo e investigar de nuevo su incidencia en los cambios.

\section{Mostrar evidencias de los resultados a la sociedad para promover el uso de buenas prácticas}

Nos parece muy necesaria la función de difusión de aquellos resultados de la práctica investigadora que por parte de los equipos se considere relevante para que puedan otros profesores tratar de incorporarlos a su actividad docente, considerando que el estado de la investigación actual del aula (tanto cualitativa como cuantitativa) debería disuadirnos de intentar cualquier transferencia directa de las conclusiones de una investigación dada como solución a problemas concretos de los docentes. Siempre va a requerir una adaptación a las circunstancias, personales y materiales, del contexto en el que se desarrolle la actividad docente.

Un reto actual es conseguir llegar a mostrar evidencias a la sociedad de los efectos educativos positivos de los entornos virtuales de enseñanza y formación. Y, en general, del uso de las TIC en la enseñanza presencial. 


\section{Reclamar ayudas para investigar}

Hay dos tipos de reclamaciones que debemos plantearnos como profesionales de la educación en nuestra faceta de investigadores. La primera es tratar de gestionar con las administraciones central y autonómica (cada colectivo de investigadores con la suya) un estatuto o protocolo que permita el acceso de los investigadores a los centros educativos públicos. En nuestra comunidad, teniendo reconocido por la propia comunidad la categoría de "grupo de investigación de excelencia" no hemos tenido toda la facilidad que hubiéramos deseado para obtener información en los centros educativos poder realizar proyectos que, en algún caso, ellos mismos no habían financiado.

La segunda, reclamar mayor esfuerzo de financiación a las administraciones central y autonómica, argumentando que en el programa marco 2020, se indica que los países de la UE deberán invertir el 3\% del PIB en I+D+i.

Estas reclamaciones responden a la necesidad, cada día más patente, de reconducir todo lo relacionado con la gestión de la investigación educativa (Tójar, 2001).

\section{A modo de síntesis}

La mejor investigación educativa, la investigación más útil será aquella que, contribuyendo a mejorar la práctica, plantee hipótesis que puedan verificarse con la propia acción educativa.

Esta investigación no puede ser otra que aquella que consiga la adecuada síntesis entre las diversas estrategias metodológicas. Parece razonable perseguir tanto el rigor como la relevancia.

Esta investigación por la que abogamos requiere:

- Mejorar el uso de las metodologías positivistas avanzadas, adecuando las técnicas a los objetivos.

- Impulsar el uso de metodologías naturalistas rigurosas (Tójar, 2006), evitando caer en la falacia del "todo vale".

- Considerar la metodología de investigación como una tecnología en la que es necesario formarse permanentemente.

- Fomentar plataformas de encuentro entre los investigadores, docentes y gestores.

- Hay que dar respuestas y soluciones a los problemas; debemos intentar favorecer el impacto social de los hallazgos relevantes.

Se podrá así, como señala Latorre (2007), contribuir a crear un conocimiento propiamente educativo: a) Útil, pertinente para manejar la complejidad del hecho educativo y para resolver las situaciones problemáticas que plantea la acción educativa (enseñanza); b) Experiencial, cargado de valor propositivo y orientado a la práctica; su construcción requiere que el educador (profesorado) reflexione sobre la experiencia profesional y personal y c) Componente clave para una acción educativa (enseñanza) de calidad, apostando por un educador (profesorado) capaz de cambiar y transformar su práctica. 
Se trata, en definitiva, de ir poniendo los cimientos para la conquista del futuro (una mejor acción educativa) que sólo será factible por una suma de calidades. Y el principal criterio de calidad de la investigación educativa debe venir dado por su contribución a esa mejora, por su utilidad.

\section{Referencias}

Alonso, P. (2005). Calidad en investigación ( $2^{\mathrm{a}}$ parte). Aproximación metodológica a las actividades de investigación. Revista de Investigación en Gestión de la Innovación y Tecnología, 33.

Bunge, M. (1980). La investigación científica. Barcelona, España: Ariel.

Bunge, M. (2003). La investigación básica. La gallina de los huevos de oro. Discurso de recepción del Doctorado Honoris Causa. Universidad de Salamanca. http://www. tindon.org/bunge.html

Campbell, D.T. y Stanley, J.C. (1978). Diseños experimentales y cuasiexperimentales en la investigación social. Buenos Aires: Amorrortu.

Carr, W. y Kemmis, S. (1988). Teoría crítica de la enseñanza. Barcelona, España: Martínez Roca.

CIDE (2001). Treinta años de investigación educativa institucional en España. Madrid, España: MEC-CIDE.

Clark, J.A. (1994). Objectivity, subjectivity and relativism in Educational Research. Curriculum Inquiry, 24, 1, 81-94.

De la Orden, A. y Mafokozi, J. (1999). La investigación educativa: Naturaleza, funciones y ambigüedad de sus relaciones con la práctica y la política educativas. Revista de Investigación Educativa, 17, 1, 7-29.

Dendaluce, I. (1988). Una reflexión metodológica sobre la investigación educativa. En I. Dendaluce (ed): Aspectos metodológicos de la investigación educativa (13-45). Madrid, España: Narcea.

Díaz Costa, E. (2009). Impacto de la investigación educativa en la práctica docente. Granada, España: Tais.

Fernández Esquinas, M. (2003). Criterios de calidad en la investigación social: la producción de datos sociales. Empiria. Revista de Metodología de Ciencias Sociales, 6, 47-77.

Gibbons, M. y otros (1997). La nueva producción del conocimiento. Barcelona, España: Pomares-Corredor.

Habermas, J. (1984). Ciencia y técnica como ideología. Madrid, España: Tecnos.

Latorre, A. (2007). Investigación-acción. Conocer y cambiar la práctica educativa. Barcelona, España: Grao.

Lewin, K. (1951). Field theory in social science. New York: Harper and Row.

Lewin, K. (1978). La teoría de campo en la ciencia social. Buenos Aires: Paidós.

MECD (2013). Objetivos educativos europeos y españoles. Estrategia educación y formación 2020. Ministerio de Educación, Cultura y Deporte. Madrid, España: MECD.

Perines, H. y Murillo, F.J. (2017). ¿Cómo mejorar la investigación educativa? Sugerencias de los docentes. Revista de la Educación Superior, 46, 181, 89-104. https://doi. org/10.1016/j.resu.2016.11.00304. 
Sabariego, M. (2004). La investigación educativa: génesis, evolución y características. En R. Bisquerra (coord.): Metodología de la Investigación Educativa (20-49). Madrid, España: La Muralla.

Stenhouse, L. (1991). Investigación y desarrollo del currículum. Madrid, España: Morata. Tejedor, F.J. (1988). El soporte estadístico en la investigación educativa. En I. Dendaluce (ed): Aspectos metodológicos de la investigación educativa (228-244). Madrid, España: Narcea.

Tejedor, F.J. (1994). La experimentación como método de investigación educativa. En V. García Hoz: Problemas y métodos de investigación en educación personalizada (256-285). Madrid, España: Rialp.

Tejedor, F.J. (2004). Investigación educativa: ¿Hacia dónde vamos? En L. Buendía, D. González y T. Pozo (Coords): Temas fundamentales en la investigación educativa (63107). Madrid, España: La Muralla.

Tejedor, F.J. (2005). Algunas reflexiones sobre el devenir de la investigación educativa a lo largo del siglo XX. En Trillo, F. Las ciencias de la educación de ayer al mañana (247-284). Servicio de Publicaciones de la Universidad de Santiago de Compostela. Santiago de Compostela, España.

Tejedor, F.J. y García-Valcárcel, A. (2012). Sociedad tecnológica e investigación educativa. Revista Española de Pedagogía, 251, 3-26.

Tójar, J.C. (2001). Planificar la investigación educativa: Una propuesta integrada. Buenos Aires: Fundec.

Tójar, J.C. (2006). Investigación cualitativa: Comprender y actuar. Madrid, España: La Muralla.

UOC (2013). Crear escuelas que preparen para el futuro. https://www.macba.cat/es/ debates-edu-hans-richard-gerver

Van Dalen, D.B. y Meyer, W.J. (1994). Manual de técnicas de investigación educativa. Buenos Aires: Paidós.

Fecha de recepción: 2 de abril de 2018

Fecha de revisión: 11 de abril de 2018

Fecha de aceptación: 23 de abril de 2018 